\title{
Number-space associations without language: Evidence from preverbal human infants and non-human animal species
}

\author{
Rosa Rugani $^{1}$ - Maria-Dolores de Hevia ${ }^{2,3}$
}

Published online: 3 August 2016

(C) Psychonomic Society, Inc. 2016

\begin{abstract}
It is well known that humans describe and think of numbers as being represented in a spatial configuration, known as the 'mental number line'. The orientation of this representation appears to depend on the direction of writing and reading habits present in a given culture (e.g., left-to-right oriented in Western cultures), which makes this factor an ideal candidate to account for the origins of the spatial representation of numbers. However, a growing number of studies have demonstrated that non-verbal subjects (preverbal infants and non-human animals) spontaneously associate numbers and space. In this review, we discuss evidence showing that preverbal infants and non-human animals associate small numerical magnitudes with short spatial extents and left-sided space, and large numerical magnitudes with long spatial extents and right-sided space. Together this evidence supports the idea that a more biologically oriented view can account for the origins of the 'mental number line'. In this paper, we discuss this alternative view and elaborate on how culture can shape a core, fundamental, number-space association.
\end{abstract}

Keywords Number $\cdot$ Mental number line $\cdot$ Space-number association $\cdot$ Infants $\cdot$ Non-human animals

Maria-Dolores de Hevia

dehevialola@gmail.com

Rosa Rugani

rosa.rugani@unipd.it

1 Department of General Psychology, University of Padova, Via Venezia, 8, Padova, Italy

2 Université Paris Descartes, Sorbonne Paris Cité, Paris, France

3 CNRS UMR 8242, Laboratoire Psychologie de la Perception, 45 rue des Saints-Pères, 75006 Paris, France

\section{Introduction}

Let's imagine the numerical interval from one to nine. Plausibly, your mental image is characterized by the visualization of numbers orderly arranged from left to right. Indeed, cognitive science has established that, for adult humans, the representations of number and space are profoundly intertwined (see de Hevia, Vallar, \& Girelli, 2006, for review). The intuition that number representation may be spatially organized dates back to 1880 , when Francis Galton first reported that humans both describe and think of numbers as being represented on diverse visuo-spatial forms, among which a horizontal continuum oriented from left (smaller numbers) to right (larger numbers): the so called mental number line (MNL; Galton, 1880, see also Restle, 1970). The first empirical evidence for a left-to-right oriented MNL was reported by Dehaene, Bossini and Giraux (1993). They presented French adults with a single Arabic digit number (from 0 to 9) in the center of a screen, and required them to press a button to indicate if the number was even and another button if the number was odd. By varying the response rule (press the left button if the number is even, or press the right button if the number is even), it was serendipitously found that responses to relatively smaller numbers (near to 0 ) were faster for the left hand and that responses to relatively larger numbers (near to 9) were faster for the right hand. This phenomenon was coined the SNARC (spatial numerical association of response codes) effect (Dehaene, Bossini, \& Giraux, 1993). It is interesting to note that, in a parity judgment task - a task in which, mathematically speaking, magnitude estimation is irrelevant-number magnitude is automatically accessed. Moreover, the effect of this spatial numerical association does not reverse in lefthanded subjects or in right-handed subjects crossing their hands. This indicates that it is the spatial correspondence between the position of the number in the MNL and the position 
in which the response is executed that explains the SNARC effect.

Therefore, the SNARC effect reflects a spatial congruency between the response side (left or right egocentric space) and the relative position of the represented numerical magnitude on a left to right oriented MNL (Dehaene et al., 1993; see also Restle, 1970). In order to test the impact of writing/reading direction, in their seminal work, Dehaene and colleagues (1993) tested this phenomenon in Iranian subjects, who had been raised in a right-to-left writing culture and then immigrated in France. Although neither a SNARC nor a reversed SNARC effects emerged in their behavior, a subsequent regression analysis indicated that the effect was larger for subjects who had been in a left-to-right culture for a longer time: the longer the time spent in France, the more the performance resembled that of native French subjects. This finding established the first basis for the suggestion that the direction of reading/writing, and therefore cultural factors, could determine the direction of the SNARC effect.

Much progress has been made since its first description, and the SNARC effect has been replicated in a number of varied experimental paradigms and in diverse populations (Patro \& Haman, 2012; Göbel, 2015; Göbel, Maier, \& Shaki, 2015; see also Fias \& Fischer, 2005; Göbel, Shaki, \& Fischer, 2011; and Dehaene, 2011). Critically, several studies support the original idea that the spatial-numerical association emerges as a result of exposure to formal instruction (Zebian, 2005; Shaki \& Fischer, 2008; Shaki, Fischer, \& Petrusic, 2009). Shaky and colleagues (2009) compared the spatial representation of numbers in three groups of human adults using a parity judgment task: Canadians, Palestinians and Israelis. Canadians, who read and write English words and Arabic numbers from left to right, showed a left-to-right mapping of numbers onto space. Palestinians, who read and write both Arabic text and Arabic-Indic numbers from right, showed an inverted spatial-numerical association (from right to left). Interestingly enough for Israelis, who read and write Hebrew words from right to left, but Arabic numbers from left to right, the association of small numbers with left space and of large numbers with right space was small and inconsistent, possibly due to the competition of opposite directionalities. These findings unambiguously support the idea that cultural conventions for reading/writing direction define the directionality (or the lack of it) of the mapping of numbers onto space (Shaki et al., 2009).

However, other studies have suggested that reading habits themselves cannot fully explain the origin of this spatialnumerical arrangement (Fischer \& Brugger, 2011). In fact, it is sufficient to change the context in which a given number has to be evaluated to invert the spatial numerical association. For instance, Bächtold and colleagues (1998) presented participants with a number comprised in the interval from 1 to 11 (without number 6). Half of the participants were instructed to imagine a ruler and to conceive each number as a distance shorter or longer than 6 . In half the trials participants had to press the leftmost key of a keyboard with the left hand to indicate a distance shorter than 6 and by pressing the rightmost key with the right hand to indicate a distance longer than 6; the response rule was inverted for the other half of trials. The other participants were instructed to imagine a clock face and to evaluate whether each number represents a time of a day earlier or later than 6 o'clock. Again, the response assignment was counterbalanced: in half of the trials they had to press the left key with the left hand to indicate a day time earlier than 6 and in the other half of trials to indicate a time later than 6. In the ruler task participants responded faster to small numbers with the left hand and to large numbers with the right hand, while in the clock-face task participants responded faster to small numbers with the right hand, and to large numbers with the left hand (Bächtold, Baumüller, \& Brugger, 1998; see also Vuilleumier, Ortigue, \& Brugger, 2004). Therefore, it is not only the culture but also the context in which a number has to be evaluated that influences its association with the left or right space, although it is possible that by 'default' (that is, in the absence of any instruction), the orientation is determined by the cultural convention in reading/writing direction. However, other studies have shown that the intensive schooling and education seems not to be essential in shaping a representation of numbers in relationship to a spatial axis. Adults who had received little or no formal education, when required to arrange numerosities on a horizontal line, showed a tendency to locate smaller numbers on the left and larger numbers on the right (Cantlon, Cordes, Libertus, \& Brannon, 2009; Dehaene, Izard, Spelke, \& Pica, 2008; Dehaene, Izard, Pica, \& Spelke, 2009). Although the specific orientation of the SNARC effect varies with culture, its presence across different cultures supports the idea that the spatial-numerical association is a universal cognitive strategy (Göbel et al., 2011). Nevertheless, it remains to be established whether the orientation of the MNL is simply modulated by educational/ cultural factors, or whether it is entirely produced by them.

The origins of the spatial-numerical association and its connections with cultural experience can hardly be conclusively demonstrated in adult humans, as it is virtually impossible to completely remove the influence of culture and language in this population. In the last decade, however, a growing number of experimental evidence has shown that the universality of the number-space association can be found in other populations beyond adult humans. Studies on pre-verbal infants and non-human animals have provided new experimental evidence supporting the idea that the number-space association would be a universal cognitive strategy that is shared by allhuman and non-human - animals (de Hevia, Girelli, \& Vallar, 2006; Rugani, Regolin, \& Vallortigara, 2007; de Hevia, Girelli, Bricolo, \& Vallar, 2008; de Hevia \& Spelke, 2009, 2010; Lourenco \& Longo, 2010; Rugani, Kelly, Szelest, 
Regolin, \& Vallortigara, 2010a; Rugani, Vallortigara, Vallini, \& Regolin, 2011a; Adachi, 2014; de Hevia, Girelli, Addabbo, \& Macchi Cassia, 2014; de Hevia, Izard, Coubart, Spelke, \& Streri, 2014; Rugani, RosaSalva, \& Regolin, 2014; Drucker \& Brannon, 2014; 2015; Bulf, de Hevia, \& Macchi-Cassia, 2015; Rugani, Vallortigara, Priftis, \& Regolin, 2015a, b). Here, we review the existing literature that supports the idea that an association of numbers with space exists independently from language. This idea, however, does not deny the importance of language and culture in shaping number-space associations, but showcases the existence of more fundamental factors other than culture and language in producing the number-space link phenomenon, and therefore considers that culture and language are critical but secondary in determining the orientation of the number-space association.

\section{Number-space association in pre-verbal human infants}

The idea that cultural artefacts determine the number-space link phenomenon was supported by the developmental literature, with the observation that children appear to show a robust, adult-like, SNARC effect only after years of schooling (Berch, Foley, Hill, \& Ryan, 1999). However, we suggested that this might be due to two factors. First, the fact that only symbolic number and relatively sophisticated tasks were used in the studies; and second, that only an oriented number-space mapping (i.e., small number associated to the left and large number to the right) was tested for, while a more basic spatial mapping of number that does not include information of spatial positions might be present earlier in development (de Hevia et al., 2008; de Hevia, Girelli, et al., 2006; de Hevia $\&$ Spelke, 2009). In fact, subsequent studies have shown that when non-symbolic number (i.e., arrays of dots) is used, the task is highly simplified, and a basic, non-oriented numberspace mapping is tested for, humans exhibit comparable number-space associations in adulthood (de Hevia et al., 2008; de Hevia, Girelli, \& Vallar, 2006; de Hevia, Vallar, \& Girelli, 2006), pre-school childhood (de Hevia \& Spelke, 2009; de Hevia, Vanderslice, \& Spelke, 2012), infancy (de Hevia \& Spelke, 2010; Lourenco \& Longo, 2010), and even at birth (de Hevia, Izard, Coubart, Spelke, \& Streri, 2014). In particular, in line bisection tasks, where participants are presented with central horizontal lines printed on a paper sheet (see Fig. 1), and are asked to quickly and accurately mark the center of the lines, adults and pre-school children show comparable spatial biases when lines are flanked by non-symbolic numerosities (dot arrays). In particular, they systematically bisect the lines towards the side ipsilateral to the larger numerosity, and this effect has been replicated across different conditions that control for non-numerical continuous variables, such as the overall area of the dot arrays, or the overall space occupied by the whole flanker (de Hevia \& Spelke, 2009). This phenomenon has been interpreted as a sort of 'cognitive' illusion by which the side of the line ipsilateral to the larger numerosity is represented as longer, and the side of the line ipsilateral to the smaller numerosity is represented as shorter. Therefore, the bisection bias towards the larger number compensates for this illusory imbalance. These studies suggest the existence of a 'non-directional' number-space mapping, one in which the information of spatial positions is not specified. These findings formed the basis for new research undertaken with even a younger population, preverbal human infants, in order to test the hypothesis that the number-space connection originates in an early-developing predisposition to relate these two dimensions.

We therefore tested preverbal infants' ability to relate the two types of information: numerical and spatial (de Hevia \& Spelke, 2010). In a first experiment, we habituated 248 month-old infants to a series of either increasing or decreasing number, while measuring their looking times to the numerical displays that changed in magnitude following a 1:2 ratio (this ratio ensures successful discrimination at this age; $\mathrm{Xu} \&$ Spelke, 2000). Once the looking time decreased significantly, signalling that habituation had been reached, we presented a series of lines lengths that differed in magnitude following the same 1:2 ratio, alternating increasing and decreasing order trials. If infants are able to transfer discrimination of the ordinal information embedded in the numerical series to the displays showing different line lengths, then infants habituated to increasing number should look longer to the lines following a decreasing order, and infants habituated to the decreasing numerical order should look longer to the series of lines following an increasing order. In fact, that was the pattern of results that we obtained: infants generalized the habituation looking pattern (low looking times) to the series of lines that followed the familiar order, and looked significantly longer to the series of lines that followed the novel order at test. Note that infants' generalization from number to length could not be based on area, as different numerosities had a constant overall area, and only different line lengths increased area with length.

A second series of experiments was aimed at extending this finding with a different method, asking also whether infants at the same age would map number and space in the absence of explicit ordinal cues. This time we presented the information of number and space at the same time, but following no predictable order (see Fig. 2). A first group of 20 infants were presented with a rule that established a positive (congruent) relationship between number and space: the larger the number, the longer the line. When attention to these displays diminished significantly, we presented infants with two new test trials: in one trial new numbers and new lines were presented following the same (positive) rule, and in a different trial new numbers and new lines were presented following a different (negative) rule, where the larger the number, the shorter the 


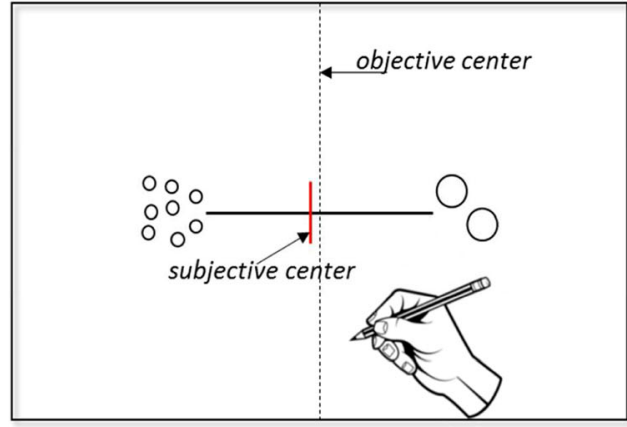

Fig. 1 In line bisection tasks, participants are asked to manually set the midpoint of a horizontal line. When using numerical flankers in the form of dot arrays (e.g., 8 vs. 2), children as well as adults show comparable biases towards the larger numerosity: when the larger numerosity is on the left side of the line, the subjective centre is biased towards the left, relative to the objective centre (left panel); when the larger number is on the right side of the line, the subjective centre is biased towards the right, relative to the objective centre (right panel). Note that in the figures the objective centre of the line is represented by the dotted line to illustrate the bias, but

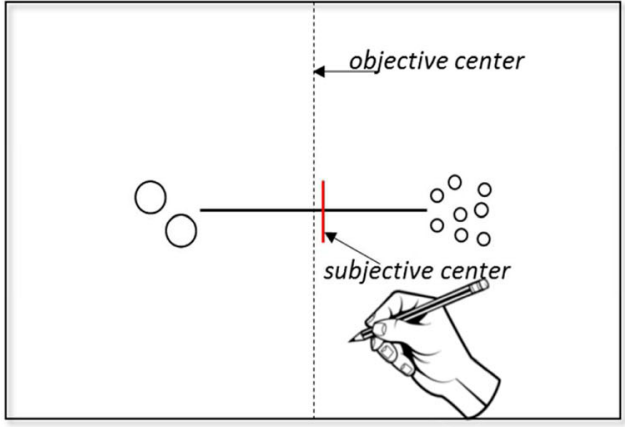

the dotted line is absent in the actual task. This finding has been interpreted as a 'cognitive' illusion of length: the side of the line with the larger numerosity is represented as longer, the side of the line with the smaller numerosity is represented as shorter, so that the bias compensates for this illusory imbalance (see de Hevia, Girelli, \& Vallar, 2006). For evidence showing that this phenomenon is not explained by an attentional bias towards the larger numerosity, see de Hevia \& Spelke (2009). Results adapted from de Hevia \& Spelke (2009)
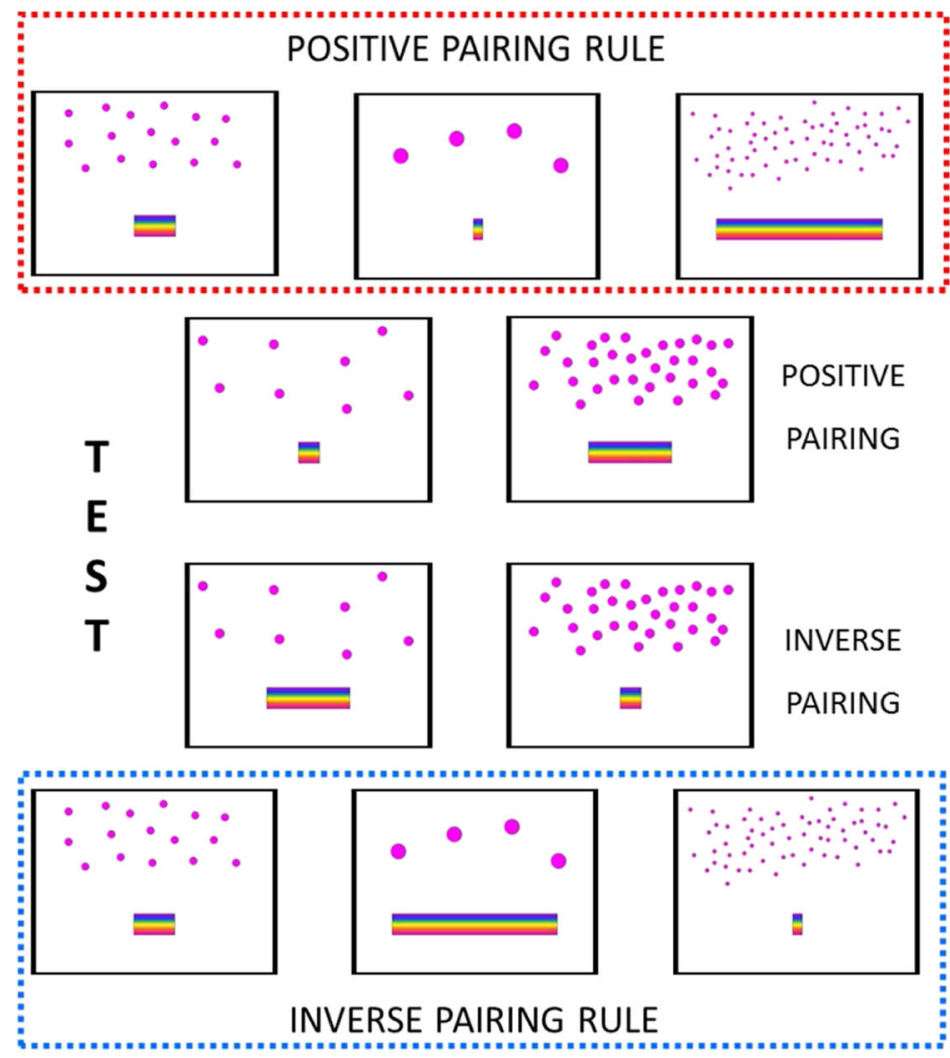
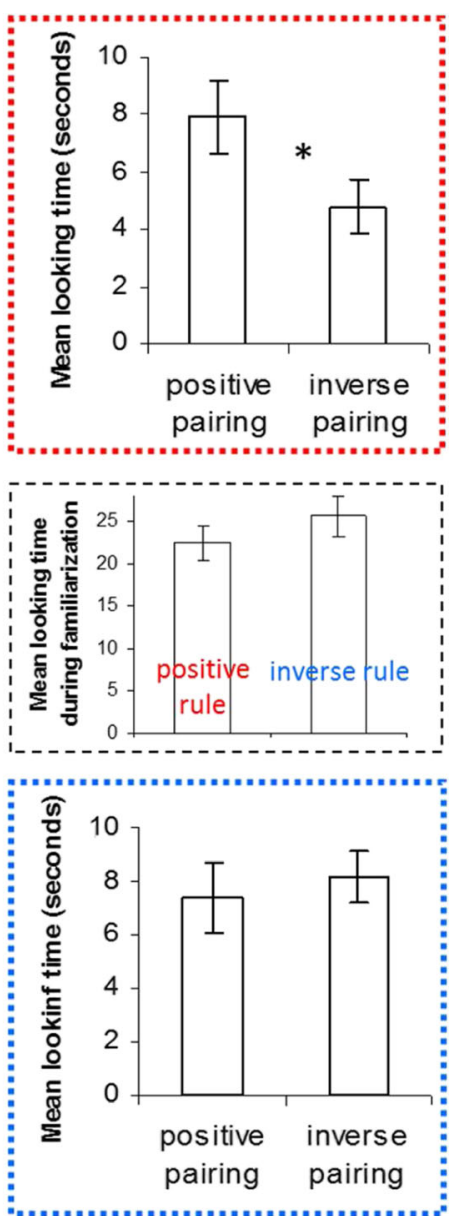

Fig. 2 Eight-month-old infants were familiarized either with a positive (red square) or with an inverse (blue square) mapping between number and line length. In the positive mapping more numerous displays are accompanied by a longer line; in the inverse mapping more numerous displays are accompanied by a shorter line. At test, all infants were presented with new mappings that followed either the positive or the inverse rule. Infants that had been familiarized with a positive pairing rule showed a significantly higher looking time for the new exemplars that followed the positive rule, while infants that had been familiarized with an inverse pairing rule did not show any looking difference at test, despite the fact that both groups of infants had received similar amounts of exposure to their respective pairing rules. The difference in performance was supported by a significant interaction between the two experiments. Adapted from de Hevia \& Spelke, (2010) 
line. We measured the looking times during the presentation of these test trials and we found a significantly higher looking time to the trial where the same (positive) rule was presented with new stimuli. Note the ability to map number and space in this and the previous experiment is revealed through contrasting patterns of looking time. In the previous experiment, when infants were habituated to an increasing or decreasing sequence of numbers, their interest in the sequence declined, so that they exhibited shorter looking times at new line lengths that exhibited the same ordinal change. In this second series of experiments, when infants were allowed to learn a specific relation between number and length from a few randomly ordered examples, they generalized the relation to new examples and showed heightened interest in new numbers and lengths that exhibited the same relation. This contrasting pattern of looking times towards novel vs. familiar test trials might indicate that the complexity of the stimuli differed across the experiments, or that congruent number-space pairings enhance attention towards them. This last possibility was in fact demonstrated in a further experiment where infants were shown an alternation of congruent and inverse numberspace pairings without being exposed a priori to any rule. Infants showed a general preference for positive pairings (see Exp. 4 in de Hevia \& Spelke, 2010). The preference for positive pairings was nonetheless more pronounced when infants were previously exposed to the positive pairing rule, suggesting that they learnt and generalized the rule when given habituated to a positive pairing rule.

Therefore, when infants are shown a positive rule between number and length they are able to learn the rule and apply it to new exemplars. However, although this finding shows that infants can learn a rule that establishes a positive relationship between number and space, it does not establish whether this link has to follow a congruent pairing, or whether infants would accept any given pairing between the two sources of information. Therefore, we tested a second group of 208 month-old infants with similar methods and materials, except that the rule shown during familiarization established a negative (inverse) relationship between number and space: the larger the number, the shorter the line. Note that this rule is equally predictable than the positive one, except that the two dimensions are paired inversely. At test, the infants in this group received the same stimuli as in the group familiarized to the positive, congruent rule. In contrast to those infants, however, in the inverse rule condition infants did not show any preference for any of the two mappings, either the positive or the inverse (see Fig. 2). This finding suggests that in the condition where they were presented with the inverse pairing, infants were not able to learn the rule and apply it to new exemplars during the test trials, as supported by the presence of a significant interaction between the test trials and the paring rule (de Hevia \& Spelke, 2010). Therefore, the mapping between number and spatial extent has a privileged structure, with larger numbers corresponding to larger spatial extents, for infants, as it does for adults (de Hevia et al., 2008; Restle, 1970).

These findings support the idea that a predisposition to relate representations of numerical magnitude to spatial length develops early in life, prior to the acquisition of language and counting, and prior to encounters with visual symbols, rulers, or other measurement devices. Similar results witnessing preverbal infants' ability to map numbers to spatial extents was provided by different researchers, and the ability to create these mappings was extended to the dimension of time. In particular, 9-month-old infants were shown to be able to generalize object properties across the dimensions of number, spatial extent, and temporal duration (Lourenco \& Longo, 2010), and generalize ordinal information from the domain of spatial extent to the domain of temporal duration (Srinivasan \& Carey, 2010). The next pressing question deriving from these findings was whether these mappings are given during the first months of life by the extensive experience with the variations of these dimensions in the environment, or whether infants have the ability to create mappings between number, space and time from birth.

We addressed these questions in a series of experiments by testing newborn infants after a few hours of life ( $7 \mathrm{~h}-96 \mathrm{~h})$ and therefore with minimal postnatal experience (de Hevia, Izard, Coubart, Spelke, \& Streri, 2014), by exploiting the evidence that human newborns represent abstract number (Izard, Sann, Spelke, \& Streri, 2009). The paradigm in our study had two phases: a familiarization phase $(60 \mathrm{~s})$ immediately followed by a test phase (see Fig. 3). Across the two phases, all infants were presented with two values of numerosities and/or durations (one small and one large), and two values of length (one small and one large). During familiarization, one of the auditory stimuli was paired with one visual length. Afterwards, during the test, the auditory numerosity and/or duration was changed (from small to large or from large to small), and was paired with the familiar and the novel visual length in two successive trials. For instance, if a baby is presented with 6 sounds and a short line, then, in test, she/he is presented with 18 sounds and a short line in one trial, and 18 sounds and a long line in another trial. Compared to familiarization, test trials thus contained either one change (auditory change only) or two changes (auditory and visual changes). Crucially, two familiarization conditions were created such that in the twochange trials the auditory numerosity/duration and visual length changed either in the same direction (either both increasing or both decreasing), or in opposite directions (one increasing, the other decreasing). The sounds used for auditory, numerical sequences were syllables repeated either 6 or 18 times (e.g., ' $\mathrm{tu}-\mathrm{tu}-\mathrm{tu}-\mathrm{tu}-\mathrm{tu}-\mathrm{tu}$ ' $v s$. ' $\mathrm{tu}-\mathrm{tu}-\mathrm{tu}-\mathrm{tu}-\mathrm{tu}-$ $\left.\mathrm{tu}-\mathrm{tu}-\mathrm{tu}-\mathrm{tu}-\mathrm{tu}-\mathrm{tu}-\mathrm{tu}-\mathrm{tu}-\mathrm{tu}-\mathrm{tu}-\mathrm{tu}-\mathrm{tu}-\mathrm{tu}^{\prime}\right)$.

We observed a significant increasing in looking time during the test trial that implemented congruent changes (same 

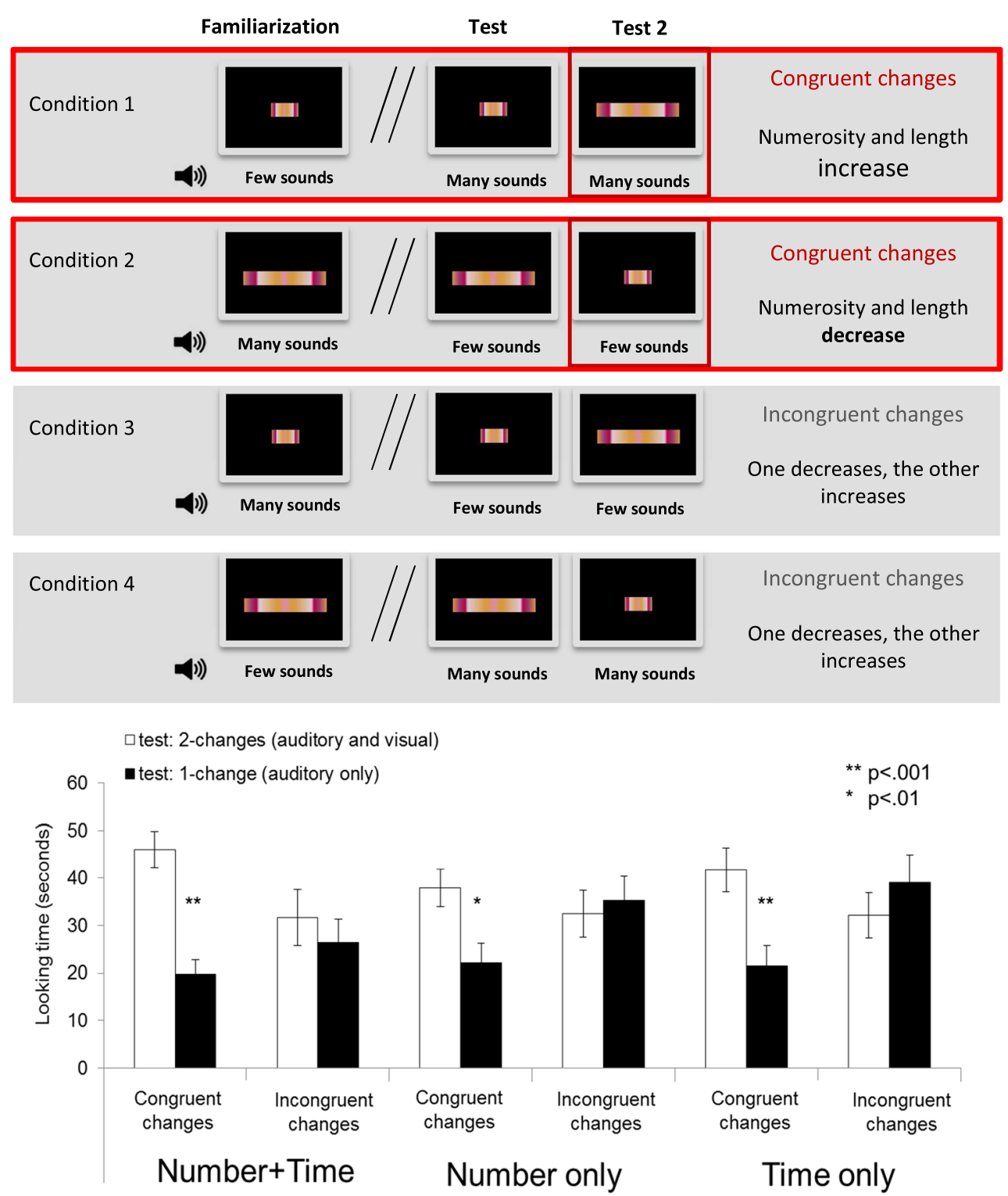

Fig. 3 Upper panel Different experimental conditions used to test newborns' ability to form mappings between auditory magnitude and visual line lengths. Each experiment (exp. 1: number+time; exp. 2: number only; exp. 3: time only) included the four conditions depicted, which consisted of a familiarization phase $(60 \mathrm{~s})$ followed by two test trials. Visual length was represented by a horizontal, coloured rectangle in the center of the screen; sounds used for auditory stimuli were sequences of syllables, repeated either 6 or 18 times (e.g., 'ga - ga - ga

direction) in magnitude across the different dimensions, relative to the test trial that did not implement these congruent changes. As we used a familiarization technique, we infer that infants 'prefer' and/or 'recognize' the stimulus with higher looking times. These findings show that human newborns are sensitive to congruent increases or decreases both of number and length, and of duration and length. In fact, they suggest that newborns build an expectation of congruency between magnitude-related changes in number, time, and space,
- ga - ga - ga'). Test trials presented either congruent or incongruent changes across the different dimensions of magnitude (auditory and visual length). Bottom panel Looking times during the two test trials where either one or two changes took place. Newborns systematically looked longer to two-change test trials provided the magnitude changes across dimensions were in the same direction (congruent changes: one increases, the other increases, or one decreases, the other decreases). Adapted from de Hevia, Izard, Coubart, Spelke, \& Streri, (2014)

from familiarization to test. Notably, neonates revealed this sensitivity both when numerical and temporal cues were simultaneously available, and when number and duration was presented in isolation. In fact, newborns were tested in three different conditions: one in which the auditory sequences that contained less syllables lasted a short time and the auditory sequences that contained more syllables lasted for a long time (in this condition both numerical and temporal cues are available in the auditory stimulus); a second condition where the 
duration of the sequences containing less syllables and the sequence containing more syllables lasted the same time (we did this by lengthening the duration of the individual syllables in the less numerous sequences, such that only the numerosity cue is available in the auditory stimulus); a third condition where we presented only one syllable at the beginning and one at the end of short and long auditory sequences (the first and last syllables were separated by a tone of variable duration, and therefore only a temporal cue was available in the auditory stimulus). In the three conditions, newborns reacted to changes in magnitude that went in the same direction but not when they varied in different directions. Taken together, these findings show that the human mind thus may be predisposed to relate these three fundamental dimensions prior to any experience with the natural correlations between numbers of objects, spatial extents, and temporal durations. Therefore, the mapping is not a mere association learnt through a statistical learning that can establish non meaningful associations, but the information of magnitude presented in the form of number, temporal duration and spatial length has to be congruent at an abstract level.

The mapping of number and space in humans is therefore functional from birth. However, this mapping does not reflect the directionality present in the adult, mature MNL, where numbers are spontaneously associated to different spatial positions (Dehaene, Bossini, \& Giraux, 1993; Restle, 1970). Recent research has started to disclose the presence of this orientation in preverbal infants' numerical processing (see Fig. 4). In particular, we have recently tested 7-month-old infants in their ability to discriminate numerical order when displacing the numerosity displays (formed by arrays of colored rectangular-shaped items) along a horizontal spatial axis (de Hevia, Girelli, Addabbo, \& Macchi-Cassia, 2014). We found that infants that are habituated to increasing or decreasing numerical sequences that are successively presented with a left-to-right orientation (e.g., small number appearing on the

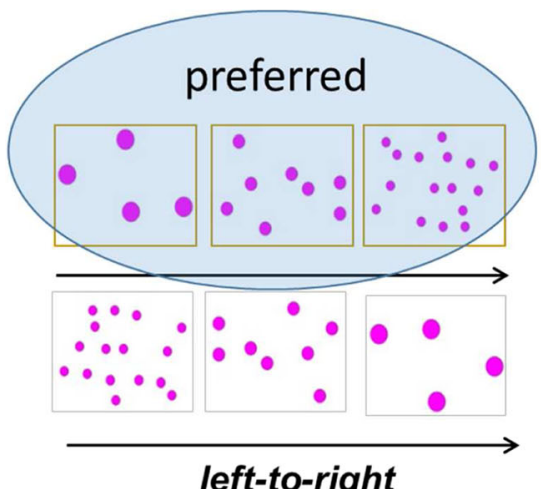

Fig. 4 When 7-month-old infants are presented with increasing or decreasing numerical sequences oriented from left-to-right, they show an ability to discriminate order, together with a preference for the increasing, left-to-right oriented sequences. When they are presented left side of the screen, medium number in the centre, and large number in the right side of the screen), show a baseline preference for increasing, left-to-right oriented numerical sequences at test, irrespective of the habituation condition they received. In fact, infants habituated to increasing numbers showed significantly longer looking times to increasing (familiar order) new numerical sequences, and infants habituated to decreasing numbers showed significantly longer times to increasing (novel order) new numerical sequences. This preference was confirmed in a further experiment that did not include a habituation phase, such that infants were presented with increasing and decreasing left-to-right oriented numerical trials in alternation. There is therefore, early in life, a privileged mapping between numbers and a spatial orientation, whereby a left-to-right orientation is preferentially associated to a series of increasing numerosities, but not to a series of decreasing numerosities. Moreover, this preference for increasing number is present only when the information is presented from left to right, but it is absent when the information is presented from right to left or even in the absence of spatial information, when the numerical displays are all presented successively in the centre of the screen (Picozzi, de Hevia, Girelli, \& Macchi Cassia, 2010). There is the possibility that the left-to-right orientation has a facilitating effect in learning or processing ordinal information; in fact, when infants of the same age received the same numerical displays but presented along a right-to-left orientation, infants failed at discriminating numerical order in both the increasing and the decreasing habituation conditions (de Hevia, Girelli, et al., 2014).

The study reviewed above provides evidence that, in the 1 st year of life, humans associate increments in numerosity with a horizontal spatial displacement from left to right, but it does not provide unambiguous support for the idea that, as adults, infants associate small numbers with the left side of space and large numbers with the right side of space (akin to a SNARC effect). Recent studies provide evidence for this

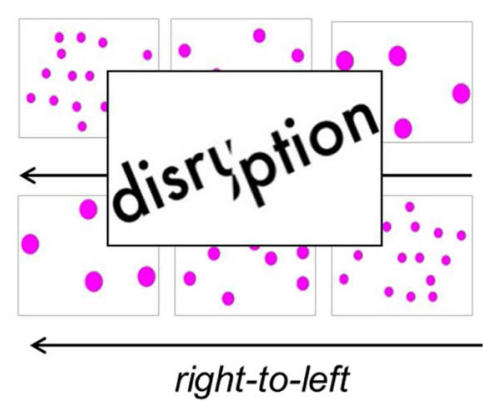

with increasing or decreasing numerical sequences oriented from rightto-left, they fail at discriminating numerical order, and therefore do not show either any preference. Results adapted from de Hevia, Girelli, Addabbo, \& Macchi Cassia (2014a) 
phenomenon. In particular, using an adaptation of the Posner paradigm, 8-month-old infants have been tested in their ability to spontaneously orient towards the left and right hemispaces when presented with incidental numerical information (see Fig. 5) (Bulf, de Hevia, \& Macchi-Cassia, 2015). We recorded infants' looking behaviour through an eye-tracking system, which allowed to track the exact position and timing of their gaze behaviour. Infants were first presented with an attention catcher in the center of the screen and, as soon as they looked at it, a non-symbolic numerosity (an array of either two or nine dots) appeared centred on the screen and was followed by either a left- or a right-lateralized target. We found that infants oriented significantly faster towards the left target when it was preceded by a small number and to the right target when it was preceded by a large number, than when the opposite combination took place (left target preceded by a large number and right target preceded by a small number).

This is the first evidence for a SNARC-like association between numbers and oriented spatial codes in preverbal infants. Moreover, this association appears to be specific for numerical information at this age and when using this type of paradigm, as the same study showed that infants do not present the same phenomenon when using small- and large-sized geometrical figures instead of numbers. Therefore, evidence that preverbal human infants in their 1st year of life create mappings that establish a correspondence between numbers and spatial extents (de Hevia, Izard, et al., 2014; de Hevia \& Spelke, 2010;

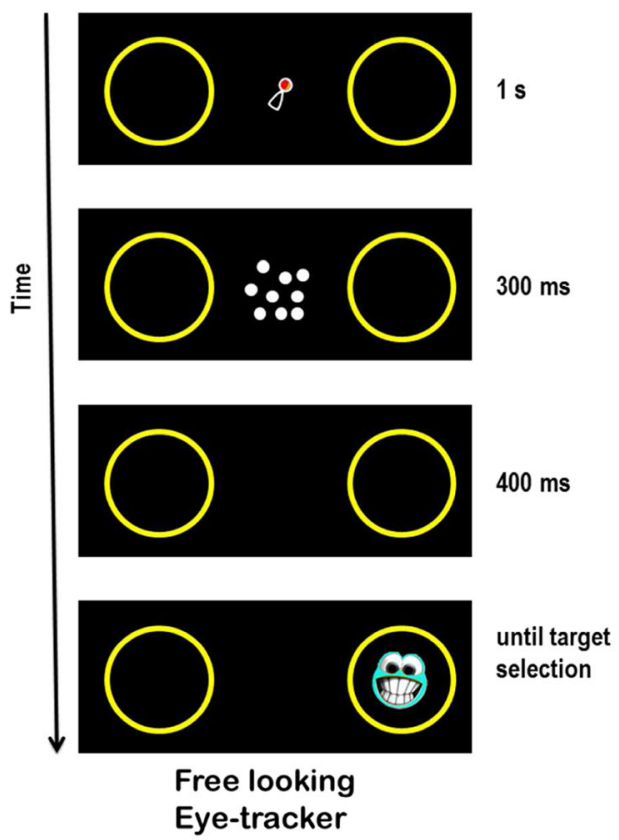

Fig. 5 Posner-like paradigm adapted to test a SNARC in infancy. Left panel shows a schematic representation of a 'congruent' trial, where a central large numerosity precedes a target appearing on the right side of the screen. Infants are faster at orienting towards the target in congruent (small numerosity in the center is followed by a target on the left side;
Lourenco \& Longo, 2010), and between numbers and spatial positions along a left-to-right oriented axis (Bulf et al., 2015; de Hevia, Girelli, et al., 2014) supports the idea that the MNL is functional from very early in development (de Hevia, Girelli, \& Macchi-Cassia, 2012), and that it does not emerge as a consequence of acquiring writing/reading abilities. Still, future studies will need to establish whether the mapping between numbers and different spatial positions is completely independent from culture in humans: during the first months of life, infants are plausibly determined in their exploration of the space by their parents' own attentional biases. However, it is still highly plausible that this mapping is in fact biologically determined in humans, as suggested by evidence demonstrating that the mapping of number onto a left-to-right oriented axis exists in nonhuman species (Drucker \& Brannon, 2014; Rugani, Kelly, Szelest, Regolin, \& Vallortigara, 2010a; Rugani, Vallortigara, Priftis, \& Regolin, 2015a). These studies will be reviewed in the next section the paper.

\section{Number space association in non-human animals}

The first evidence that suggests a predisposition to "count from left to right" in non-human animals was obtained investigating ordinal ability in domestic chicks. In their seminal work Rugani, Regolin and Vallortigara (2007) wanted to study whether day-old animals could master an ordinal task that

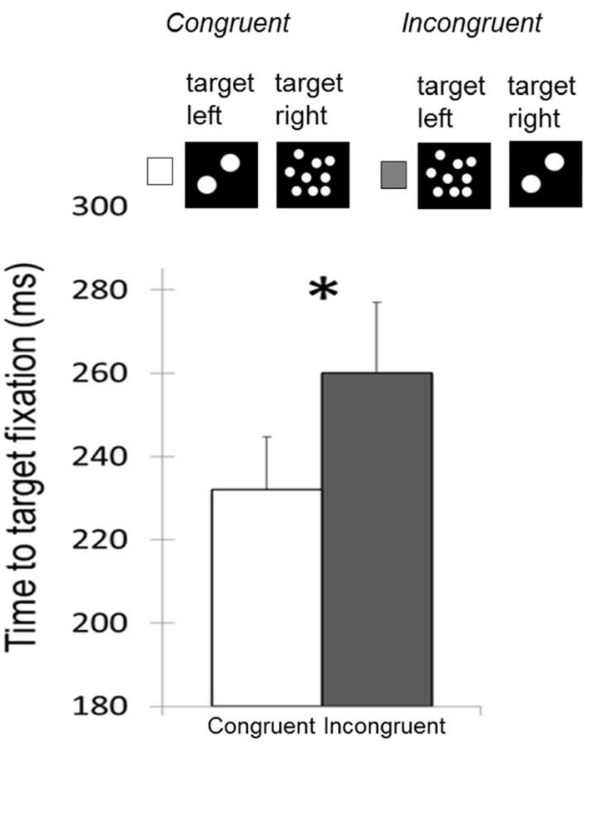

large numerosity in the centre is followed by a target on the right side) than in incongruent (small numerosity in the center is followed by a target on the right side; large numerosity in the centre is followed by a target on the left side) trials. Adapted from Bulf, de Hevia, \& Macchi Cassia (2015) 
consisted in identifying a target element (e.g., the fourth) in a series of identical elements on the basis of its ordinal position. Five-day-old chicks were trained to peck a target element for food reinforcement. For different groups of chicks the target was the third, the fourth or the sixth, in a series of ten identical, equidistant fixed and sagittally (with respect to the chicks' starting position) aligned elements (see Fig. 6a). All groups of animals mastered the task, pecking at test significantly above chance solely the target element, while all others were pecked at or below chance. This suggested that chicks could encode serial-ordinal information. Nevertheless, because the position of the elements remained identical during training and during testing, correct responses could have been based both on numerical as well as on spatial cues (e.g., distances from the ends of the series or from the starting position). To eliminate these alternative explanations, in one of the control experiments, a new group of chicks was trained to identify the fourth element in a series of ten identical, equidistant, and sagittally aligned elements, which during training were maintained in fixed positions. At test the series was maintained identical to that used during training, but it was rotated by $90^{\circ}$, and therefore fronto-parallelly oriented with respect to the bird's starting position (see Fig. 6b). In this way, two were the possible correct choices: the target element from the left and the one from the right end. Chicks pecked statistically above chance the 4th element from the left end of the series rather than the one from the right end (Rugani, Regolin, \& Vallortigara, 2007).

This lateral bias has been also replicated in subsequent studies. One of these studied the ordinal abilities of two bird species: day-old domestic chicks, and adult Clark's nutcrackers. Separate groups of birds learned to peck, for food reinforcement, at either the fourth or the sixth element in a series of 16 identical, fixed and equidistant elements, sagittally aligned with respect to the birds' starting position. At frontoparallel test, when the series was maintained identical but rotated by $90^{\circ}$, both species pecked at the target element from the left end but not the one from the right end (Rugani, Kelly, Szelest, Regolin, \& Vallortigara, 2010a). As the authors argued, this bias resembles the human tendency to "count" from left to right. Nevertheless, other interpretations could explain these results. The asymmetry in birds' behavior (i.e., the preference for targets located in the left hemispace) may depend, at least in part, on a specific bias in the allocation of attention in extra-corporeal space, somewhat comparable to human "pseudoneglect" (Albert, 1973). "Pseudoneglect" phenomena have been indeed found in birds, which appear to overestimate the size of the left side of space in line bisection tasks (Regolin, 2006), and with a selective allocation of attention to the left hemifield during free foraging (Diekamp, Regolin, Vallortigara, \& Güntürkün, 2005). Alternatively, the leftsided preference could be due to a possible asymmetry related to the availability of certain kind of spatial cues. In fact, being the series used during training and during fronto-parallel test identical, the target in both experimental phases was located at the same distance from the two ends of the series. In the chick forebrain the spatial information is represented in the right hemisphere (Tommasi \& Vallortigara, 2001; Tommasi, Gagliardo, Andrew, \& Vallortigara, 2003; Regolin, Garzotto, Rugani, Pagni, \& Vallortigara, 2005; Rashid \& Andrew, 1989; reviewed by Vallortigara, Chiandetti, Sovrano, Rugani, \& Regolin, 2010a; Vallortigara, Regolin, Chiandetti, \& Rugani, 2010b; Rugani, Vallortigara, \& Regolin, 2015c). The possible use of spatial information would therefore produce a right hemisphere dominance. This would favor an allocation of attention into the left hemispace, thus generating the bias to "count" from left to right. To test whether the possible use of this kind of spatial information could affect the left-bias, the possible use

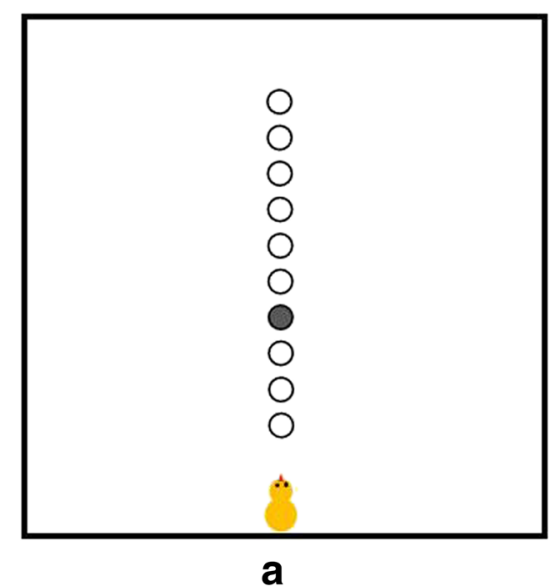

Fig. 6 a Schematic representation of the apparatus, with the chick in the starting position, and the sagittal disposition of the elements used for training and testing. During training and testing all elements were identical; here the filled circle indicates the element reinforced during training. b Schematic representation of the apparatus, with the chick in

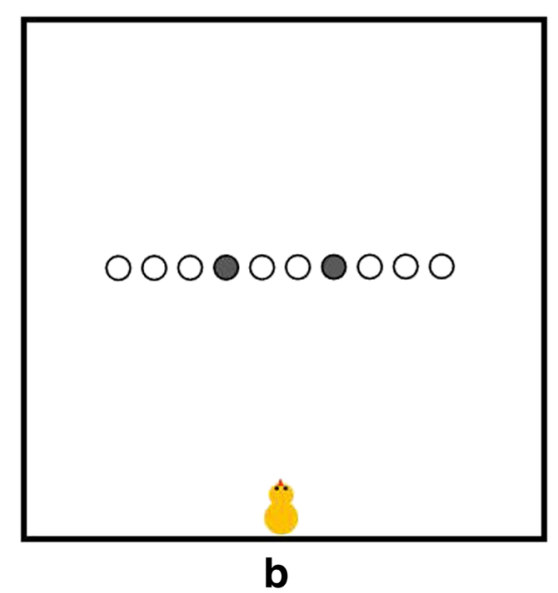

the starting position, and the fronto-parallel disposition of the elements used for testing. During training and testing all elements were identical; here the filled circles indicate the two possible correct positions. Adapted from Rugani, R., Regolin, L. and Vallortigara, G. (2007) 
of the spatial information, during training and/or during testing, was prevented in a set of consecutive experiments.

The aim of a first experiment was to disentangle which information (either the spatial or the numerical one) could be used by day-old chicks to identify the target during frontoparallel test. A new group of chicks of the same age were trained to identify the fourth element in a series of ten fixed and equidistant elements (See Fig. 7a). In this way, the target could be identified using both spatial and numerical cues. During the fronto-parallel test, the inter-element distances were manipulated in order to create a conflict between spatial and numerical information. For a group of chicks the interelement distances were enlarged, so that in the new frontoparallel series the third element occupied the position previously occupied by the fourth element (See Fig. 7b). For another group of birds, during the fronto-parallel test the interelement distance was reduced, so that the fifth element occupied the position previously occupied by the fourth element (See Fig. 7c).

Even in this case, both groups of chicks clearly selected the target's ordinal position, neglecting the element (the third or the 5th) located at the correct distance from the beginning of the series (Rugani, Vallortigara, Vallini, \& Regolin, 2011). This demonstrates that chicks preferentially rely on numerical rather than on spatial information. Interestingly enough chicks, in this conflicting fronto-parallel test, pecked statistically above chance both the target elements: the fourth from the left and the 4th from the right.

In a subsequent experiment chicks were trained to identify the 4 th element in a series of 10 elements solely on the basis of its serial order (Rugani et al., 2011a). To avoid any possible use of the spatial information (neither in terms of absolute nor relative distances among the elements of the series), the interelement distances were systematically changed from trial to trial during training and fronto-parallel test. Again, chicks were able to identify the target element, but again their pecks were identically directed on the fourth left and on the fourth right element. This indicates that day-old birds can identify an ordinal position solely on the basis of numerical information. Moreover these data, together with the data of the previous experiment, suggest that is the combining use of spatial and numerical information that would determine the left-bias. Alternatively, the absence of bias in this latter fronto-parallel test could be due to a novelty effect, produced by a variation (non-specifically numerical) that birds experienced during testing. To test this hypothesis, a new group of chicks were trained to peck the fourth element in a series of ten elements, maintained in fixed position, and aligned sagittally with respect to the bird's starting position. During the fronto-parallel test, the inter-element distance along the series was maintained identical, but the color of the elements was changed. For example, a new group of subjects were trained with a series of red elements, and then they were tested with a series of green elements, and vice versa for the other group of animals. Chicks pecked solely the fourth left element above chance, while the fourth right element was pecked at chance. This means that a non-spatial change does not affect the presence of the left-bias.

Overall, this finding suggests that, in a serial ordinal task, chicks preferentially rely on numerical cues and not on the non-numerical cue. Nevertheless, even when the spatial information seemed to be not used, its availability affected chicks' behavior: solely when both spatial and numerical information could be learned during training and later used during testing, chicks showed a left-bias (Rugani et al., 2011a). Rugani and colleagues (2011a) suggested that purely numerical information could be represented bilaterally in the left and right cerebral hemisphere, while spatial information would be represented unilaterally in the right hemisphere.

It has been argued that asymmetrical processing of spatial and numerical information could be present only in the avian brain, in which visual information is elaborated separately by the two hemispheres (Drucker \& Brannon, 2014). Birds with laterally placed eyes, in fact, present a complete decussation of

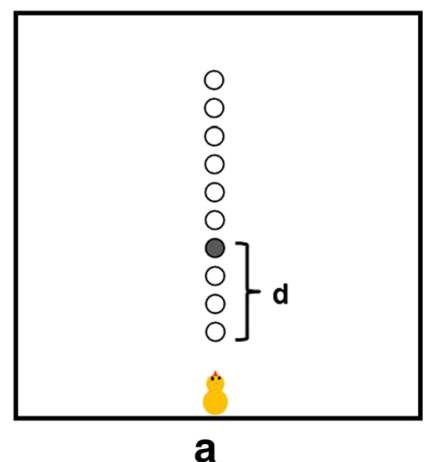

a

Fig. 7 a Schematic representation of the apparatus and the disposition of the series of elements used for training. During training all elements were identical, here the filled circles indicates the element reinforced during training. The distance of the correct element from the closest end of the series (d) was maintained identical throughout training. b Schematic

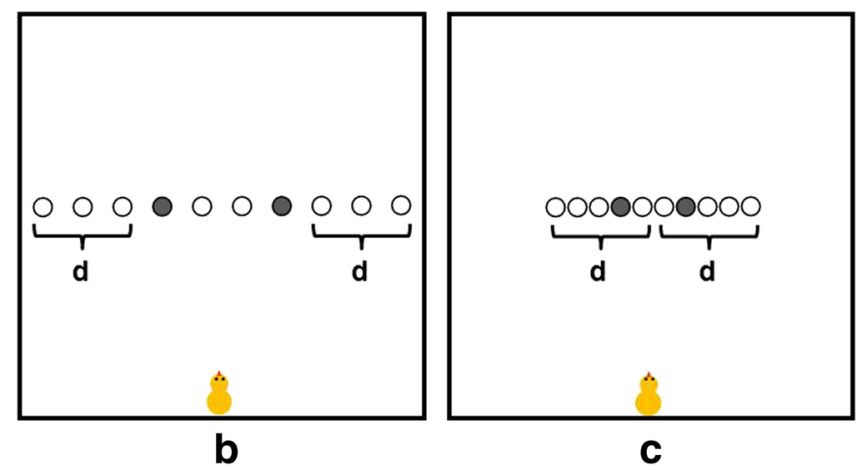

representation of the fronto-parallel series in the condition in which the inter-element distances were enlarged. c Schematic representation of the fronto-parallel series in the condition in which the inter-element distances were reduced. Adapted from Rugani, R., Vallortigara, G., Vallini, B. and Regolin, L. (2011a) 
fibres at the optic chiasm (Weidner et al. 1985; Ocklenburg \& Güntürkün, 2012), and lack of a structure homologous to the corpus callosum (even though other smaller tracts allow inter hemispheric communication, e.g. Hardy et al., 1984; Robert \& Cunéond, 1969; Theiss et al. 2003; Zeier \& Karten, 1973). Thus, each eye projects mainly to the visual system of the contralateral hemisphere (Deng and Rogers, 1998), and, consequently, visual information from one side of the space is elaborated mainly in the contralateral hemisphere (the information provided by the left eye is elaborated mainly by the right hemisphere, and the information provided by the right eye is elaborated mainly by the left hemisphere). This strong lateralization underlies various behavioural asymmetries observed in birds (Daisley, Mascalzoni, Rosa Salva, Rugani, \& Regolin, 2009; Rogers, Vallortigara, \& Andrews, 2013), and it could possibly underlie also the asymmetry found in the spatial-ordinal task (Drucker \& Brannon, 2014). Nevertheless the asymmetric encoding of the order of items starting from the left side of space has also been found in primates (Drucker \& Brannon, 2014). Recently, Drucker and Brannon (2014) developed a computer version of the serialordinal task that allows a very precise control of the appearance and of the spatial disposition of the stimuli. Monkeys were trained to touch the fourth item from the bottom in a series of five identical and vertically aligned items presented on a touch screen. Then, on probe trials, when the series was rotated by $90^{\circ}$, monkeys preferentially touch the fourth element from the left. Interestingly, the left bias emerged also when, using a series of five elements, the fourth left element was located on the right hemifield (Drucker \& Brannon 2014).

Overall, the demonstration of a spatial numerical association in three different species indicates that asymmetrical processing could not be a prerogative only of the lateralized avian brain, but is rather a more common strategy.

Further studies, employing a different task, found a facilitation in ordering numbers from left to right in non-human animals. Adachi (2014) trained adult chimpanzees to touch, in ascendant order, Arabic numerals (1-9) shown in random locations on a touch screen. At test, only two numerals (1 and 9) were presented on the screen, displayed horizontally, one on the left and the other on the right side. Chimpanzees responded faster when " 1 " was located on the left side and "9" on the right side, and slower when "9" was located on the left and " 1 " on the right (Adachi, 2014). The facilitation on the " 1 on the left -9 on the right" condition indicates that chimpanzees map a learned sequence onto space (Adachi, 2014). This study, as well previous evidence, showing that animals manifest a bias in processing ordinal information from left to right, supports the idea that number-space association occurs in absence of language.

Recently, it has been shown that day-old domestic chicks spontaneously (in absence of specific numerical training) associated a large number with the right space (Rugani, Rosa-Salva, \& Regolin, 2014). In this study, the motivation of day-old chicks to follow the larger group of artificial rearing companions (objects chicks have been familiarized with through exposure and that are treated by these birds as social companions, Regolin, Garzotto, Rugani, \& Vallortigara, 2005; Regolin, Rugani, Pagni, \& Vallortigara 2005b; Rugani, Fontanari, Simoni, Regolin, \& Vallortigara, 2009; Rugani, Regolin, \& Vallortigara 2010b, 2011b; Rugani, Cavazzana, Vallortigara, \& Regolin, 2013; Fontanari, Rugani, Regolin, \& Vallortigara, 2011; 2014) was analyzed to investigate whether the performance, in dealing with an arithmetic task, would be affected by the left-right position of the two sets. The idea was that, if smaller and larger numbers are differently associated with left and right space, then a better performance (higher number of correct responses) would be expected whenever chicks were required to respond to the larger group on the right.

Soon after hatching, chicks were reared with a group of identical objects (bi-dimensional red squares). On day 4, chicks underwent a free-choice test. During testing chicks, confined in a starting box, faced two identical opaque screens, one on their left and the other on their right. From their starting box, birds could see, throughout a transparent partition, some objects, identical to the rearing ones, disappearing, one by one, behind either screen (e.g., five objects were consecutively hidden behind a screen and ten behind the other). The screen hiding the larger set was randomized between testing trials, so that the higher number of objects could be hidden half of the time behind the left screen and the other half of the time behind the right screen. It should be noted that, in this kind of test, the performance of chicks, even when statistically above chance, does not reach $100 \%$ of correct responses, even when both screens hide small numbers and simple comparisons (Rugani, Fontanari, Simoni, Regolin, \& Vallortigara, 2009; Rugani, Cavazzana, Vallortigara, \&Regolin, 2013). This is principally due to three reasons: the very young age of the subjects, the fact that they have not undergone any numerical training, and especially to the presence behind the two screens of positive stimuli. As a consequence, even if in a few trials, circumnavigation of the screen hiding the smaller group of artificial social companions is plausible, this will be significantly above chance solely if animals can discriminate between the two groups. In both the numerical comparisons considered ( 5 vs. 10 and 6 vs. 9) chicks performed better when the higher number of objects was hidden behind the screen on their right (Rugani, Rosa-Salva, \& Regolin, 2014). This asymmetry could be due to a specific effect of numerical processing, or rather to a non-numerical preference when searching for social attractors on the right side. In a subsequent experiment, to control for this possibility, the same number of objects (two) was hidden behind the left and the right screen. If the bias that emerged in the previous experiment was non- 
numerical in nature, it would have appeared also in this comparison. Nevertheless, when the two screens hid the same number of objects (2 vs. 2 ), chicks did not reveal any directional bias. Future experiments are needed to disentangle whether these results can also be obtained when comparing two large numbers of objects, such as 5 vs. 5 or 10 vs. 10 . Nevertheless, it seems that a right-sided bias can be observed only when chicks had to choose between sets differing in number. Such a finding would fit with the hypothesis that large numbers are associated with right space.

Overall, these studies suggest that a predisposition to associate numbers onto space could be rooted in a non-linguistic basis, indicating that culture could play a secondary role in determining number-space association. Nevertheless, it should be noted that all these studies showed only partial effects. In fact, even if all these investigations support the existence of a spatial numerical association in non-human animals, they all fail to demonstrate, using the same paradigm, an association of small numbers with the left space and of large numbers with the right space. The ordinal paradigm has allowed it to be demonstrated that, in identifying a target on the basis of its serial-ordinal position, domestic chicks, Clark's nutcrackers and rhesus monkeys show a left bias. Both birds (trained to select the fourth element from the beginning of the series) and monkeys (trained to select the fourth element from the "bottom" end of the series) responded to the fourth left element whenever, at test, the series was rotated by $90^{\circ}$. Caroline Drucker and Elizabeth Brannon (2014) affirmed that it was not clear if monkeys selected the target element considering it as the fourth element from the bottom end of the series or as the second from the top end. Also, the selection of the fourth left element at test would fit with the two alternative hypothesis, but, independently of the bottom or top strategy, monkeys' behavior shows a tendency to associate number with space. If monkeys used the bottom strategy, identifying the target element as the fourth one from the bottom end of the series, their choice for the fourth left element, would indicate a left bias in orientation of attention in the fronto-parallel test. Alternatively, if monkeys used the top strategy, identifying the target as the second from the right, their choice for the fourth element from the left, that could be also considered as the second from the right, would indicate a right bias. In any case, whichever strategy the animals may have used supports the spatial numerical association hypothesis.

A study by Rugani, Rosa-Salva and Regolin (2014) demonstrated an association of large number with right side. It is undoubtedly interesting that a left or a right bias emerged in different numerical tasks. Nevertheless, also in this case, the employment of this paradigm showed only one sole direction of the spatial-numerical association. Due to the paradigm employed, and considering that this was based on the spontaneous tendency of chicks to follow the larger number of artificial social companions, it is hard to motivate birds to select the smaller number of objects, which would be the condition necessary to demonstrate an association of small numbers with the left space. The same partial evidence was also reported by Adachi (2014), who demonstrated a facilitation to touch the numbers $1-9$ in ascending order solely when the 1 was on the left and the 9 on the right. It would be fascinating to investigate whether animals trained to touch numbers in descending order would show a facilitation in touching the numbers $9-1$ when the 9 is on the right and the 1 on the left. Moreover, it should be noted that, in Adachi's paradigm, it is not clear if chimpanzees computed the magnitude values of the Arabic numbers or, alternatively, if they responded to a sequential learning task. In other words, did chimpanzees learn that the magnitude of numeral 9 is larger than that of numeral 8 ? Or did they merely learn that the symbol "9" went after the symbol "8"? To reply to this objection, it would be crucial to replicate Adachi's paradigm using non-symbolic stimuli that convey the magnitude cues, e.g., using arrays composed by different numbers of dots.

Last but not least all the previously discussed works fail to demonstrate a fundamental characteristic of human numberspace mapping: the relativity to the considered numerical interval. To give an example, in a " $1-9$ " interval, the number 9 is associated with the right space, but, in the interval "9-18" the same number 9 is associate with the left space (Dehaene, 2011). A more convincing demonstration of spatial numerical association in non-human animals would need to demonstrate, employing the same paradigm, three aspects: (1) an association of small numbers with the left space, (2) an association of large numbers with the right space, and (3) the relativity of this spatial numerical association. To date, only a single study has proved the relativity of the spatial-numerical association in non-human animals. A more recent work has shown that dayold chicks associate relatively small numbers with left space and relatively large numbers with right space (Rugani, Vallortigara, Priftis, \& Regolin, 2015a, b). Three-day old domestic chicks were trained to find a food reward behind a panel by circumnavigating it. The panel depicted a certain number of little squares, e.g., five. Then, at test, chicks were faced with a pair of identical panels, placed one aside the other, both depicting the same number of elements smaller or larger than the one used during training (in this case either two in the Small Number Test, or eight in the Large Number Test).

When, in the Small Number Test, the pair of panels depicted two squares each, the animals searched behind the left panel, while they went behind the right one when panels depicted eight squares, in the Large Number Test.

Other chicks, trained with a 20 -squares-panel, were placed in front of pairs of panels with either 8 or 32 squares. To guess where the reward could be (as during testing chicks never find the reward behind either panel), in the first case ( 8 vs. 8 , Small Number Test), they went behind the left panel, while in the 
second case ( 32 vs. 32 Large Number Test) they went behind the right one. Thus, the same number of squares " 8 " produced a different behavior in animals trained with 5 (i.e., going to the right) or with 20 elements (i.e., going to the left). Chicks chose the right or the left side of their visual space, respectively, depending on the numbers seen at test being greater or smaller than the number seen at training. This recalls the relativity of the human MNL. Nevertheless, in these experiments, the elements used in both the training and testing stimuli were all identical red squares of the same dimensions. Because quantitative information (i.e., area, perimeter, etc.) of a group of elements co-varied with the number of elements that composed the group, the association could be due to a numerical as well as to quantitative information. In order to exclude any possible use of quantitative information, three different controls were conducted. In all control conditions, the target number was " 20 " and the testing numbers were " 8 " ( 8 vs. 8 in the Small Number Test) and " 32 " (32 vs. 32 in the Large Number Test). In the first condition, heterogeneous elements, differing from one another in shape, color and size, were used. In the second condition, the stimuli were controlled for the overall area, which consisted of the sum of the areas of all elements depicted in each stimulus. In a third condition, a contemporary control for multiple quantitative cues was performed. This condition indeed controlled: the overall perimeter (the summation of the perimeters of all elements depicted in each stimulus), density (the mean distance among the elements), and occupancy (the overall space occupied by each group of elements). More importantly, in the third condition, there was a negative correlation between overall area and number: the overall area of the 32 elements was smaller than that of the 20 elements (training), and smaller than that of 8 elements. Again, chicks circumnavigate the left panel in the Small Number Test $(8 \mathrm{vs} .8)$ and the right panel in the Large Number Test (32vs.32), demonstrating that is the number of elements - and not the quantity of area, perimeter, color, light, density - that is associated with space (Rugani, Vallortigara, Priftis, \& Regolin, 2015a; 2015b). It should also be noted that, besides some critical comments, these results can be explained by the numerical magnitudes of the stimuli and not by aesthetic judgments, nor by biological or behavioral brain asymmetries (Rugani, Vallortigara, Priftis, \& Regolin, 2016a; 2016b).

Overall, these results indicate that a disposition to associate numerical magnitudes onto a left-to-right oriented space does exist in non-human, non-linguistic species. Even more impressively, number-space association is observed in animals with very reduced numerical experience, supporting a nativistic foundation of such association. Because the non-verbal numerical cognition is shared by all animals (Cantlon \& Brannon 2007; Dehaene 2011), we suspect that a similar predisposition is embodied in the architecture of all animal neural systems responding to numbers, including humans.

\section{Conclusions}

In this review, we provide a thorough description of the evidence supporting spatial-numerical associations in preverbal infants and non-human subjects. Overall, the studies presented here advocate a reconsideration of the relationship between numerical abilities and verbal language, especially mature forms of numerical representation such as the MNL. Taken together, these studies suggest that language is not a prerequisite for determining the origins of the spatialnumerical association. As in the behavioral sciences, the simplest explanations should be considered first, and, only when these are discarded experimentally, should explanations involving more sophisticated abilities be considered; the work reviewed here suggests that language is not necessary for the development of a rudimentary comprehension of numerical magnitudes (Bulf et al., 2015; Stancher, Rugani, Regolin, \& Vallortigara, 2015; de Hevia, Girelli, et al., 2014; de Hevia, Izard, et al., 2014; Miletto Petrazzini, Agrillo, Piffer, \& Bisazza 2014; Stancher, Sovrano, Potrich, \& Vallortigara, 2013; de Hevia, Girelli et al., 2012; de Hevia \& Spelke, 2010; Lourenco \& Longo, 2010; Vallortigara, et al., 2010a, b; Carey, 2009; Izard et al., 2009; Xu, Spelke, \& Gottard, 2005; Spelke, 2000), nor for the association of numbers to space (Bulf et al., 2015; Brugger, 2015; Rugani, et al., 2015a, b, c; de Hevia, Girelli, et al., 2014; de Hevia, Izard, et al., 2014; McCrink and Opfer, 2014; Rugani et al., 2014, 2011a, 2010a; de Hevia \& Spelke, 2010; Lourenco \& Longo, 2010; Rugani, et al., 2007). We do not intend to deny the fundamental role that cultural factors, such as the direction of reading/writing, play in shaping the numberspace association (de Hevia et al. 2012). The effect of reading/writing direction in affecting the spatial-numerical association has been shown unambiguously. For instance, as the study by Zebian (2005) demonstrates, human adults for whom Arabic is the main language show an inverted SNARC effect, and, as the study by Shaki, Fischer and Petrusic (2009) demonstrates, human adults with mixed reading habits (i.e., those brought up reading both left to right and right to left) show no SNARC at all (see "Introduction" section). Moreover, a recent study on 3and 4-year-old children has shown that factors other than language might affect the number-space mapping. In particular, a brief $(\approx 15 \mathrm{~min})$ non-numerical, spatial movement training (either left-to-right or right-to-left oriented) is sufficient to influence the direction of the number-space association in pre-literate 3- to 4-year-old children (Patro, Fischer, Nuerk, \& Cress, 2016). The authors proposed the 'Implicit Assumption Account', whereby an early preference for spatial arrangements of number magnitudes arises as an effect of implicit learning about culturespecific scanning of the objects in the environment, which 
can be observed in children before they start to read and write (Patro et al., 2016).

The studies reported here, testing non-verbal subjects with different paradigms and at difference ages, demonstrate convincingly that the association of numbers with space is functional in non-verbal and pre-verbal subjects. By integrating all the existing evidence, we believe the following picture takes form: the spatial-numerical association is a common characteristic of the non-verbal "core system of number", shared by all animals (humans included, for a review see, Spelke, 2000; Vallortigara, 2012; Rugani et al., 2015c), that somehow facilitates exploration of increasing magnitudes from left to right. On these basis, the influence of culture and experience modulates the orientation of this association (de Hevia, Girelli, \& Macchi Cassia, 2012). In this context, it is remarkable that, independently of its direction, the association of number and space seems to be a universal strategy (Gobel, Shaki, \& Fischer, 2011).

The challenge at present is to explain the origins of this phenomenon. It has been proposed that hemispheric brain asymmetries could be the basis of number-space association (de Hevia, Girelli, \& Macchi Cassia, 2012; de Hevia, Girelli, et al., 2014; Rugani, et al., 2015c). Brain asymmetry is indeed a common and ancient trait of vertebrates, which would have helped animals to process different kinds of information within a specialized (dominant) hemisphere. Although the specific direction of hemispheric specialization may have been, in principle, totally arbitrary, in humans, as well as in the domestic chicken, a right hemisphere dominance is found in visuospatial and/or numerical/quantitative tasks (Emerson \& Cantlon, 2015; Rugani, et al., 2015a, c; Hyde, Boas, Blair, \& Carey 2010; Rugani, Vallortigara, \& Regolin, 2016c; see also Vallortigara, 2012). The predominant role of the right hemisphere in both visuo-spatial tasks and numerical ordering, biases initial attention to the left side of both physical space and number space; therefore, the tendency to start to "count" from left to right, to prefer increasing, left-to-right oriented numerical sequences, and to associate 'small' with the left side of space and 'large' with the right side of space.

Another possibility is that space, number and time would be considered as part of a more general magnitude system, as proposed by Walsh (2003) in the ATOM theory (A Theory Of Magnitude). Walsh (2003) proposed that the neuroanatomical basis of this magnitude system could be localized in neurons of the inferior parietal cortex - an area that is activated in the elaboration of spatial, numerical and temporal information (Harvey, Fracasso, Petridou, \& Dumoulin, 2015; Simon, 2002; Rao, Mayer, \& Harrington, 2001; Cochon, et al., 1999; Dehaene et al., 1999). Due to the common neural basis, spatial, numerical and temporal information would be interconnected. The ATOM theory would explain not only the SNARC (Spatial-Numerical Association of Response Codes) effect, but also the SQUARC (Spatial-Quantity Association of Response Codes) effect (Walsh, 2003). Adult humans in fact associate magnitudes such as volume of liquid (Kirjakovski \& Utsuki, 2012), pitch height (Rusconi, Kwan, Giordano, Umiltà, \& Butterworth, 2006; Pitteri, Marchetti, Priftis, \& Grassi, 2016), music tempo (Prpic, Fumarola, de Tommaso, Baldassi, \& Agostini, 2013), and temporal duration (Vallesi et al., 2008; Bonato, Zorzi, \& Umiltà, 2012) with an oriented space. Even if the association of non-numerical magnitudes with space has been demonstrated only in adult humans to date, it is plausible that similar mechanisms of mapping magnitude onto space could be present also in non-human animals and in human infants. However, studies with infants and pre-school children suggest that the association between numbers, temporal durations, and spatial extent is privileged among mappings with other quantitative dimensions, such as brightness or loudness. In fact, infants can transfer discrimination from number to length (de Hevia \& Spelke, 2010; Lourenco \& Longo, 2010), number and time (Lourenco \& Longo, 2010), and length and time (Lourenco \& Longo, 2010; Srinivasan \& Carey, 2010), but fail to transfer from number to brightness (Harvey et al. 2013), and from loudness to length (Srinivasan \& Carey, 2010). Similarly, pre-school children readily create mappings between number and length but fail at creating number-brightness associations (de Hevia et al. 2012). Hubbard and colleagues (2005) have suggested that numerical-spatial interactions would arise from common parietal circuits for attention to external space and internal representations of numbers. This would explain why the elaboration of numerical and spatial information is profoundly intertwined (Drucker \& Brannon, 2015).

A third possibility could be that numerical representations are spatially organized in the brain. In humans, populations of neurons tuned to small numbers that are topographically organized have been described in the parietal cortex (Harvey et al., 2013), as well as similarly topographically organized maps responding to object size (or length) that largely overlap with numerosity maps and show correlated tuning preferences (Harvey, Fracasso, Petridou, \& Dumoulin, 2015). It has been suggested that this neural organization could determine the organization of magnitudes along the MNL (see Drucker \& Brannon, 2015). Although such a topographical representation has not been found in non-human animals, electrophysiological studies have shown that, in the macaque brain, neurons tuned to numbers are located in the intra-parietal cortices (Nieder \& Miller, 2004), and that some of these neurons can be tuned to both number and length (Tudusciuc \& Nieder, 2007), although their topographical organization has not been defined. Recently, neurons selective to numbers have been found in an endbrain 
association area (nidopallium caudolaterale, NCL) of crows (Ditz \& Nieder, 2015). Ditz and Nieder (2015) recorded the activity of single neurons of endbrain of crows while they were solving a numerical matching-tosample task. Birds were first presented with an array of dots (one to five). Then, a second array of dots, depicting the same or a different numbers of dots, appeared. Crows were trained to peck the second display if the number of dots depicted in the two arrays were identical. Comparisons of both behavioral and neural tuning are best described on a logarithmic number line (Ditz \& Nieder, 2015). Because non-verbal numerical cognition is shared by many animal species (Vallortigara, 2012), it is plausible that a similar map of neurons tuned to numbers would be a common characteristic of the architecture of animal neural systems.

The complete available scenario describing the origins of the spatial-numerical association is still complex and controversial. On the one hand, it has been shown that language can influence, and therefore determine, the directionality of the spatial-numerical association; on the other hand, it has been shown that a spatial-numerical association is present in both pre-verbal and non-verbal subjects. The role of culture (instantiated in directional spatial biases) might eventually strengthen or counteract a biological bias (de Hevia et al., 2012). In fact, people who read from right to left show opposite spatial associations to those who read from left to right (Dehaene et al., 1993; Shaki et al. 2009). Studies testing numerical-spatial associations across development and across cultures are needed for a complete picture of both the origins and developmental course of these associations. Moreover, the specific directionality of the number-space association can be determined by experiences not limited to writing or reading (see Patro et al., 2016). This apparently contradicts the idea that spatial biases for numbers are driven by ancient factors in brain organization. However, this evidence might be taken as suggestive of the plasticity of the numerical-spatial associations' phenomenon. In fact, culture also rapidly modifies core biological attentional biases, as shown by the pseudoneglect phenomenon, which exhibits an opposite direction depending on the culture (i.e., slight left-sided bias in left-to-right reading cultures, and slight right-sided bias in right-to-left reading cultures, see Chokron \& Imbert, 1993). Similarly, a basic small-left and large-right association might be modulated rapidly by idiosyncratic visuo-spatial biases. Nevertheless, it is important to note that, although the specific orientation of the MNL varies with the influence of cultural conventions, its presence across all cultures suggests that the spatial-numerical association is a universal cognitive strategy (Göbel, Shaki, \& Fischer, 2011).

The tendency to associate magnitude with space is not limited only to numbers but it present also with volume of liquid (Kirjakovski \& Utsuki, 2012), pitch height (Rusconi, Kwan, Giordano, Umiltà, \& Butterworth, 2006; Pitteri, Marchetti, priftis, \& Grassi, 2016), music tempo (Prpic, Fumarola, de Tommaso, Baldassi, \& Agostini, 2013), and temporal duration (Vallesi et al., 2008; Bonato, Zorzi, \& Umiltà, 2012). This may reflect the fact that, in some way, still to be determined, animal brains are organized to associate quantities and space. This may be due to the fact that, in a natural environment, continuous and numerical cues usually co-vary. From this perspective, it is not surprising that the brain may possess a system to represent both discrete and continuous quantities via the same symbolic currency.

As it stands, we strongly believe that, instead of driving the efforts to support one or the other line of evidence or not, the most relevant step in understanding a complex phenomenon such as spatial-numerical association, will be reached once the theory allows us to comprehend this phenomenon in its whole complexity, without simplifying all the existing, and apparently contradictory, evidence. This is an enterprise we consider both exciting and challenging.

Acknowledgments MDdH was supported by a research grant from Fondation Fyssen, and by the French National Agency for Research (ANR-15-CE28-0003; project acronym 'NUMSPA').

\section{References}

Adachi, I. (2014). Spontaneous spatial mapping of learned sequence in chimpanzees: Evidence for a SNARC-like effect. PLoS One, 9, e90373. doi:10.1371/journal.pone.0090373

Albert, M. L. (1973). A simple test of visual neglect. Neurology, 23(6), 658-664.

Bächtold, D., Baumüller, M., \& Brugger, P. (1998). Stimulus-response compatibility in representational space. Neuropsychologia, 36, 731735. doi:10.1016/S0028-3932(98)00002-5

Berch, D. B., Foley, E. J., Hill, R. J., \& Ryan, P. M. (1999). Extracting parity and magnitude from Arabic numerals: Developmental changes in number processing and mental representation. Journal of Experimental Child Psychology, 74(4), 286-308.

Bonato, M., Zorzi, M., \& Umiltà, C. (2012). When time is space: Evidence for a mental time line. Neuroscience \& Biobehavioral Reviews, 36, 2257-2273.

Brugger, P. (2015). Chicks with a number sense. Science, 347(6221), 477-478. doi:10.1126/science.aaa4854

Bulf, H., de Hevia, M. D., \& Macchi-Cassia, V. (2015). Small on the left, large on the right: Numbers orient preverbal infants' visual attention onto space. Developmental Science. doi:10.1111/desc.12315

Cantlon, J. F., \& Brannon, E. M. (2007). How much does number matter to a monkey? (Macaca mulatta). Journal of Experimental Psychology: Animal Behaviour Processes 33(1), 32-41. doi:10.1037/0097-7403.33.1.32

Cantlon, J. F., Cordes, S., Libertus, M. E., \& Brannon, E. M. (2009). Comment on 'Log or Linear? distinct intuitions of the number scale in Western and Indigene cultures'. Science, 323, 38b. doi:10.1126 /science.1164773

Carey, S. (2009). The origin of concepts. New York: Oxford University Press.

Chokron, S., \& Imbert, M. (1993). Influence of reading habits on line bisection. Cognitive Brain Research, 1(4), 219-222.

Cochon, F., Cohen, L., van de Moortele, P. F., \& Dehaene, S. (1999). Differential contributions of the left and right inferior parietal 
lobules to number processing. Journal of Cognitive Neuroscience, 11, 617-630.

Daisley, J. N., Mascalzoni, E., Rosa Salva, O., Rugani, R., \& Regolin, L. (2009). Lateralization of social cognition in the domestic chicken (Gallus gallus). Philosophical Transactions of the Royal Society of London - B, 364, 965-981. doi:10.1098/rstb.2008.0229

de Hevia, M. D., Girelli, L., Addabbo, M., \& Macchi Cassia, V. (2014). Human infants' preference for left-to-right oriented increasing numerical sequences. PLoS One, 9(5), e96412. doi:10.1371/journal. pone. 0096412

de Hevia, M. D., Girelli, L., Bricolo, E., \& Vallar, G. (2008). The representational space of numerical magnitude: Illusions of length. The Quarterly Journal of Experimental Psychology, 61, 1496-1514. doi:10.1080/17470210701560674

de Hevia, M. D., Girelli, L., \& Vallar, G. (2006). Numbers and space: A cognitive illusion? Experimental Brain Research, 168, 254-264. doi: $10.1007 / \mathrm{s} 00221-005-0084-0$

de Hevia, M. D., Izard, V., Coubart, A., Spelke, E. S., \& Streri, A. (2014). Representations of space, time, and number in neonates. Proceedings of the National Academy of Science of the United States of America, 111(13), 4809-4813. doi:10.1073 /pnas.1323628111

de Hevia, M. D., \& Spelke, E. S. (2009). Spontaneous mapping of number and space in adults and young children. Cognition, 110, 198207. doi:10.1016/j.cognition.2008.11.003

de Hevia, M. D., \& Spelke, E. S. (2010). Number-space mapping in human infants. Psychological Science, 21(5), 653-660. doi: $10.1177 / 0956797610366091$

de Hevia, M., Vallar, G., \& Girelli, L. (2006). Visuo-spatial components of numerical representation. Advances in Consciousness Research, 66, 155.

de Hevia, M. D., Vanderslice, M., \& Spelke, E. S. (2012). Crossdimensional mapping of number, length and brightness by preschool children. PLoS ONE, 7(4), e35530. doi:10.1371/journal. pone. 0035530

Dehaene, S. (2011). The Number Sense: How the Mind Creates Mathematics, Revised and Updated. New York: Oxford University Press.

Dehaene, S., Bossini, S., \& Giraux, P. (1993). The mental representation of parity and number magnitude. Journal of Experimental Psychology: General, 122(3), 371-396. doi:10.1037/00963445.122.3.371

Dehaene, S., Izard, V., Pica, P., \& Spelke, E. S. (2009). Response to comment on "Log or linear? Distinct intuitions of the number scale in Western and Amazonian indigene cultures". Science, 323, 38. doi:10.1126/science.1164878

Dehaene, S., Izard, V., Spelke, E. S., \& Pica, P. (2008). Log or linear? Distinct intuitions of the number scale in western and amazonian indigene cultures. Science, 320, 1217-1220. doi:10.1126 /science. 1156540

Dehaene, S., Spelke, E. S., Pinel, P., Stanescu, R., \& Tsivkin, S. (1999). Sources of mathematical thinking: Behavioural and brain imaging evidence. Science, 284, 970-974.

Deng, C., \& Rogers, L. J. (1998). Bilaterally projecting neurons in the two visual pathways of chicks. Brain Research, 794, 281-290.

Diekamp, B., Regolin, L., Güntürkün, O., \& Vallortigara, G. (2005). A left-sided visuospatial bias in birds. Current Biology, 15, R372R373.

Ditz, H. M., \& Nieder, A. (2015). Neurons selective to the number of visual items in the corvid songbird endbrain. Proceedings of the National Academy of Sciences of the United States of America, 112(25), 7827-7832. doi:10.1073/pnas. 1504245112

Drucker, C. B., \& Brannon, E. M. (2014). Rhesus monkeys (Macaca mulatta) map number onto space. Cognition, 132, 57-67.
Drucker, C. B., \& Brannon, E. M. (2015). Commentary on: Numberspace mapping in the newborn chick resembles humans' mental number line. Front Psychol, 6, 352. doi:10.3389/fpsyg.2015.00352

Emerson, R. W., \& Cantlon, J. F. (2015). Continuity and change in children's longitudinal neural responses to numbers. Developmental Science, 18(2), 314-326.

Fias, W., \& Fischer, M. H. (2005). Spatial representation of number. In J. Campbell (Ed.), Handbook of mathematical cognition (pp. 43-54). New York: Psychology Press.

Fischer, M. H., \& Brugger, P. (2011). When digits help digits: Spatialnumerical associations point to finger counting as prime example of embodied cognition. Frontiers in Psychology, 2, 260. doi:10.3389 /fpsyg.2011.00260

Fontanari, L., Rugani, R., Regolin, L., \& Vallortigara, G. (2011). Object individuation in three-day old chicks: Use of property and spatiotemporal information. Developmental Science, 14(5), 1235-1244. doi:10.1111/j.1467-7687.2011.01074.x

Fontanari, L., Rugani, R., Regolin, L., \& Vallortigara, G. (2014). Use of kind information for object individuation in young domestic chicks. Animal Cognition, 17(4), 925-935. doi:10.1007/s10071-013-0725-9

Galton, F. (1880). Visualised numerals. Nature, 21, 252-256. doi: $10.1038 / 021252 \mathrm{a} 0$

Göbel, S. M. (2015). Up or down? Reading direction influences vertical counting direction in the horizontal plane - a cross-cultural comparison. Frontiers in Psychology, 6, 228. doi:10.3389 /fpsyg.2015.00228

Göbel, S. M., Maier, C. A., \& Shaki, S. (2015). Which numbers do you have in mind? Number generation is influenced by reading direction. Cognitive Processing, 16, 241-244. doi:10.1007/s10339-0150715-8

Göbel, S. M., Shaki, S., \& Fischer, M. H. (2011). The cultural number line: A review of cultural and linguistic influences on the development of number processing. Journal of Cross-Cultural Psychology, 42, 543-565. doi:10.1177/0022022111406251

Hardy, O., Leresche, N., \& Jassik-Gerschenfeld, D. (1984). Postsynaptic potentials in neurons of the pigeon's optic tectum in response to afferent stimulation from the retina and other visual structures. Brain Research, 311, 65-67. doi:10.1016 /0006-8993(84)91399-4

Harvey, B. M., Fracasso, A., Petridou, N., \& Dumoulin, S. O. (2015). Topographic representations of object size and relationships with numerosity reveal generalized quantity processing in human parietal cortex. Proceedings of the National Academy of Sciences of the United States of America, 112(44), 13525-13530. doi:10.1073/pnas

Harvey, B. M., Klein, B. P., Petridou, N., \& Dumoulin, S. O. (2013). Topographic representation of numerosity in the human parietal cortex. Science, 341(6150), 1123-1126. doi:10.1126/science.1239052

Hubbard, E. M., Piazza, M., Pinel, P., \& Dehaene, S. (2005). Interactions between number and space in parietal cortex. Nature Reviews Neuroscience, 6(6), 435-448.

Hyde, D. C., Boas, D. A., Blair, C., \& Carey, S. (2010). Near-infrared spectroscopy shows right parietal specialization for number in preverbal infants. Neuroimage, 53(2), 647-652. doi:10.1016/j. neuroimage.2010.06.030

Izard, V., Sann, C., Spelke, E. S., \& Streri, A. (2009). Newborn infants perceive abstract numbers. Proceedings of the National Academy of Sciences of the United States of America, 106(25), 10382-10385. doi:10.1073/pnas.0812142106

Kirjakovski, A., \& Utsuki, N. (2012). From SNARC to SQUARC: Universal mental quantity line? International Journal of Psychological Studies, 4, 217-227.

Lourenco, S. F., \& Longo, M. R. (2010). General magnitude representation in human infants. Psychological Science, 21, 873-881.

McCrink, K., \& Opfer, J. E. (2014). Development of spatial-numerical associations. Current Directions in Psychological Science, 23(6), 439-445. 
Miletto Petrazzini, M. E., Agrillo, C., Piffer, L., \& Bisazza, A. (2014). Ontogeny of the capacity to compare discrete quantities in fish. Developmental psychobiology, 56(3), 529-536. doi:10.1002 dev.21122

Nieder, A., \& Miller, E. K. (2004). A parieto-frontal network for visual numerical information in the monkey. Proceedings of the National Academy of Sciences of the United States of America, 101(19), $7457-7462$

Ocklenburg, S., \& Güntürkün, O. (2012). Hemispheric asymmetries: The comparative view. Frontiers in Psychology, 3, 1-9. doi:10.3389 /fpsyg.2012.00005

Patro, K., Fischer, U., Nuerk, H. K., \& Cress, U. (2016). How to rapidly construct a spatial-numerical representation in preliterate children (at least temporarily). Developmental Science, 19(1), 126-144. doi:10.1111/desc.12296

Patro, K., \& Haman, M. (2012). The spatial-numerical congruity effect in preschoolers. Journal of Experimental Child Psychology, 111, 534 542

Picozzi, M., de Hevia, M. D., Girelli, L., \& Macchi Cassia, V. (2010). Seven-month-old infants detect ordinal numerical relationships within temporal sequences. Journal of Experimental Child Psychology, 107, 359-367.

Pitteri, M., Marchetti, M., Priftis, K., \& Grassi, M. (2016). Naturally together: Pitch-height and brightness as coupled factors for eliciting the SMARC effect in non-musicians. Psychological Research. doi:10.1007/s00426-015-0713-6

Prpic, V., Fumarola, A., de Tommaso, M., Baldassi, G., \& Agostini, T. (2013). A SNARC-like effect for music tempo. Review in Psychology, 20, 47-51.

Rao, S. M., Mayer A. M., \& Harrington D. L. (2001). The evolution of brain activation during temporal processing. Nature Neuroscience, 4, 317-323.

Rashid, N., \& Andrew, R. J. (1989). Right hemisphere advantages for topographical orientation in the domestic chick. Neuropsychologia, 27, 937-948. doi:10.1016/0028-3932(89)90069-9

Regolin, L. (2006). The case of the line-bisection: When both humans and chickens wander left. Cortex, 42, 101-103. doi:10.1016/S00109452(08)70330-7

Regolin, L., Garzotto, B., Rugani, R., \& Vallortigara, G. (2005). Working memory in the chick: Parallel and lateralized mechanisms for encoding of object- and position- specific information. Behavioural Brain Research, 157, 19. doi:10.1016/j. bbr.2004.06.012

Regolin, L., Rugani, R., Pagni, P., \& Vallortigara, G. (2005). Delayed search for a social and a non-social goal object by the young domestic chick (Gallus gallus). Animal Behaviour, 70, 855-864. doi:10.1016/j.anbehav.2005.01.014

Restle, F. (1970). Speed of adding and comparing numbers. Journal of Experimental Psychology, 83, 274-278.

Robert, F., \& Cuénod, M. (1969). Electrophysiology of the intertectal commissures in the pigeon. I. Analysis of the pathways. Experimental Brain Research, 9, 116-122.

Rogers, L. J., Vallortigara, G., \& Andrew, R. J. (2013). Divided brains: The biology and behavior of brain asymmetries. Cambridge: Cambridge University Press.

Rugani, R., Cavazzana, A., Vallortigara, G., \& Regolin, L. (2013). One, two, three, four, or is there something more? Numerical discrimination in day-old domestic chicks. Animal Cognition, 16, 557-564. doi:10.1007/s10071-012-0593-8

Rugani, R., Fontanari, L., Simoni, E., Regolin, L., \& Vallortigara, G. (2009). Arithmetic in newborn chicks. Proceedings of the Royal Society B, 276, 2451-2460. doi:10.1098 /rspb.2009.0044

Rugani, R., Kelly, M. D., Szelest, I., Regolin, L., \& Vallortigara, G. (2010a). Is it only humans that count from left to right? Biology Letters, 6, 290-292. doi:10.1098/rsb1.2009.0960
Rugani, R., Regolin, L., \& Vallortigara, G. (2007). Rudimental numerical competence in 5-day-old domestic chicks: Identification of ordinal position. Journal of Experimental Psychology: Animal Behaviour Processes, 33(1), 21-31. doi:10.1037/0097-7403.33.1.21

Rugani, R., Regolin, L., \& Vallortigara, G. (2010b). Imprinted numbers: Newborn chicks' sensitivity to number vs. continuous extent of objects they have been reared with. Developmental Science, 13(5), 790-797. doi:10.1111/j.1467-7687.2009.0

Rugani, R., Regolin, L., \& Vallortigara, G. (2011b). Summation of large numerousness by newborn chicks. Frontiers of Comparative Psychology, 2, 179. doi:10.3389/fpsyg.2011.00179

Rugani, R., RosaSalva, O., \& Regolin, L. (2014). Lateralized mechanisms for encoding of object. Behavioral evidence from an animal model: The domestic chick (Gallus gallus) Frontiers of Comparative Psychology, 5, 150. doi:10.3389/fpsyg.2014.00150

Rugani, R., Vallortigara, G., Priftis, K., \& Regolin, L. (2015a). Numberspace mapping in the newborn chick resembles humans' mental number line. Science, 347, 534-536. doi:10.1126/science.aaa1379

Rugani, R., Vallortigara, G., Priftis, K., \& Regolin, L. (2015b). Comments to" Number-space mapping in the newborn chick resembles humans' mental number line. Science, 348, 1438.

Rugani, R., Vallortigara, G., Priftis, K., \& Regolin, L. (2016a). Piece of evidence. Commentary: Ancestral mental number lines: What is the evidence? Frontiers in. Psychology, 7, 553. doi:10.3389 /fpsyg.2016.00553

Rugani, R., Vallortigara, G., Priftis, K., \& Regolin, L. (2016b). Response: Newborn chicks need no number tricks. Commentary: Numberspace mapping in the newborn chick resembles humans' mental number line. Frontiers in Human Neuroscience, 10, 31. doi:10.3389/fnhum.2016.00031

Rugani, R., Vallortigara, G., Vallini, B., \& Regolin, L. (2011a). Asymmetrical number-space mapping in the avian brain. Neurobiology of Learning and Memory, 95, 231-238. doi:10.1016 jj.nlm.2010.11.012

Rugani, R., Vallortigara, G., \& Regolin, L. (2015c). At the root of the leftright asymmetries in spatial numerical processing: From domestic chicks to human subjects. Journal of Cognitive Psychology, 27(4), 388-399. doi:10.1080/20445911.2014.941846

Rugani, R., Vallortigara, G., \& Regolin, L. (2016c). Mapping number to space in the two hemispheres of the avian brain. Neurobiology of Learning and Memory, 133, 13-18. doi:10.1016/j.nlm.2016.05.010

Rusconi, E., Kwan, B., Giordano, B., Umiltà, C., \& Butterworth, B. (2006). Spatial representation of pitch height: The SMARC effect. Cognition, 99, 113-129.

Shaki, S., \& Fischer, M. (2008). Reading space into numbers-a crosslinguistic comparison of the SNARC effect. Cognition, 108(2), 590-599. doi:10.1016/j.cognition.2008.04.001

Shaki, S., Fischer, M. H., \& Petrusic, W. M. (2009). Reading habits for both words and numbers contribute to the SNARC effect. Psychonomic Bulletin \& Review, 16(2), 328-331. doi:10.3758 /PBR.16.2.328

Simon, O., Mangin, J. F., Cohen, L., Le Bihan, D., \& Dehaene, S. (2002). Topographical layout of hand, eye, calculation and language-related areas in the human parietal lobe. Neuron, 33, 475-487.

Spelke, E. S. (2000). Core knowledge. American Psychologist, 55, 1233 1243. doi:10.1037/0003-066X.55.11.1233

Srinivasan, M., \& Carey, S. (2010). The long and the short of it: On the nature and origin of functional overlap between representations of space and time. Cognition, 116(2), 217-241. doi:10.1016/j. cognition.2010.05.005

Stancher, G., Rugani, R., Regolin, L., \& Vallortigara, G. (2015). Numerical discrimination by frogs (Bombina orientalis). Animal Cognition, 18(3), 605-616. doi:10.1007/s10071-014-0791-7

Stancher, G., Sovrano, A. V., Potrich, D., \& Vallortigara, G. (2013). Discrimination of small quantities by fish (redtail splitfin, 
Xenotoca eiseni). Animal Cognition, 16, 307-312. doi:10.1007 /s10071-012-0590-y

Theiss, M. P. H., Hellmann, B., \& Güntürkün, O. (2003). The architecture of an inhibitory sidepath within the avian tectofugal system. NeuroReport, 14, 879-882.

Tommasi, L., Gagliardo, A., Anrew, R. J., \& Vallortigara, G. (2003). Separate processing mechanisms for encoding of geometric and landmark information in the avian hippocampus. European Journal of Neuroscience, 17, 1695-1702.

Tommasi, L., \& Vallortigara, G. (2001). Encoding of geometric and landmark information in the left and right hemisphere of the avian brain. Behavioural Neuroscience, 115, 602-613. doi:10.1037/07357044.115.3.602

Tudusciuc, O., \& Nieder, A. (2007). Neuronal population coding of continuous and discrete quantity in the primate posterior parietal cortex. Proceedings of the National Academy of Sciencesof the United States of America, 104(36), 14513-14518.

Vallesi, A., Binns, M. A., \& Shallice, T. (2008). An effect of spatialtemporal association of response codes: Understanding the cognitive representations of time. Cognition, 107, 501-527. doi:10.1016/j. cognition.2007.10.011

Vallortigara, G. (2012). Core knowledge of object, number, and geometry: A comparative and neural approach. Cognitive Neuropsychology, 29, 213-236. doi:10.1080/02643294.2012.654772

Vallortigara, G., Chiandetti, C., Sovrano, V. A., Rugani, R., \& Regolin, L. (2010a). Animal Cognition. Wiley Interdisciplinary Reviews: Cognitive Science, 1, 882-893. doi:10.1002/wcs.75
Vallortigara, G., Regolin, L., Chiandetti, C., \& Rugani, R. (2010b). Rudiments of mind: Number and space cognition in animals. Cognition \& Behavior Reviews, 5, 78-99. doi:10.3819 /ccbr.2010.50004

Vuilleumier, P., Ortigue, S., \& Brugger, P. (2004). The number space and neglect. Cortex, 40(2), 399-410. doi:10.1016/s0010-9452(08 70134-5

Walsh, V. (2003). A theory of magnitude: Common cortical metrics of time, space and quantity. Trends in Cognitive Science, 7(11), 483-488.

Weidner, C., Reperant, J., Miceli, D., Haby, M., \& Rio, J. P. (1985). An anatomical study of ipsilateral retinal projections in the quail using radioautographic, horseradish peroxide, fluorescence and degeneration techniques. Brain Research, 340, 99-108.

Xu, F., \& Spelke, E. S. (2000). Large number discrimination in 6-monthold infants. Cognition, 74(1), B1-B11.

Xu, F., Spelke, E. S., \& Goddard, S. (2005). Number sense in human infants. Trends in Cognitive Science, 2(3), 87-97.

Zebian, S. (2005). Linkages between number concepts, spatial thinking, and directionality of writing: The SNARC effect and the reverse SNARC effect in English and Arabic monoliterates, biliterates, and illiterate Arabic speakers. Journal of Cognition and Culture, 5(1), 165-190. doi:10.1163/1568537054068660

Zeier, H. J., \& Karten, H. J. (1973). Connections of the anterior commissure in the pigeon (Columba livia). Journal of Comparative Neurology, 150, 201-216. 